\title{
Decomposable Penalty Method for Generalized Game Problems with Joint Constraints
}

\author{
I. V. Konnov1
}

\begin{abstract}
We consider an extension of a noncooperative game problem where players have joint binding constraints. In this case, justification of a generalized equilibrium point needs a reasonable mechanism for attaining this state. We suggest to combine a penalty method together with shares allocation of right-hand sides, which replaces the initial problem with a sequence of the usual Nash equilibrium problems together with an upper level variational inequality as a master problem. We show convergence of solutions of these auxiliary penalized problems to a solution of the initial game problem under weak coercivity conditions.
\end{abstract}

Key words: Noncooperative games, joint constraints, generalized equilibrium points, decomposable penalty method, variational inequality.

\section{Introduction}

Noncooperative games with joint (binding) constraints date back to early works [1][5] and these problems are investigated in many recent works; see e.g. 6] -8 ] and the references therein. Let us consider the generalized l-person noncooperative game, where the $i$-th player has its particular strategy set $X_{i} \subseteq \mathbb{R}^{n_{i}}$ and a payoff (utility) function $f_{i}: X \rightarrow \mathbb{R}$ with

$$
X=X_{1} \times \ldots \times X_{l}, n=\sum_{i=1}^{l} n_{i} .
$$

Besides, all the players together with the above utility functions and strategy sets have the joint constraint set

$$
Y=\left\{\begin{array}{l|l}
x \in \mathbb{R}^{n} & \sum_{i=1}^{l} h_{i}\left(x_{i}\right) \leq b
\end{array}\right\},
$$

where $x=\left(x_{1}, \ldots, x_{l}\right)^{\top}, h_{i}\left(x_{i}\right)=\left(h_{i 1}\left(x_{i}\right), \ldots, h_{i m}\left(x_{i}\right)\right)^{\top}, h_{i j}: \mathbb{R}^{n_{i}} \rightarrow \mathbb{R}, j=1, \ldots, m$, $i=1, \ldots, l$ are given functions, $b \in \mathbb{R}^{m}$ is a fixed vector in $\mathbb{R}^{m}$, as an addition to the

\footnotetext{
${ }^{1}$ Department of System Analysis and Information Technologies, Kazan Federal University, ul. Kremlevskaya, 18, Kazan 420008, Russia.

E-mail: konn-igor@ya.ru
} 
set $X$. That is, they have the common feasible set

$$
D=X \bigcap Y
$$

A point $x^{*}=\left(x_{1}^{*}, \ldots, x_{l}^{*}\right)^{\top} \in D$ is said to be an equilibrium point for this game, if

$$
f_{i}\left(x_{-i}^{*}, y_{i}\right) \leq f_{i}\left(x^{*}\right) \quad \forall\left(x_{-i}^{*}, y_{i}\right) \in D, i=1, \ldots, l ;
$$

where we set $\left(x_{-i}, y_{i}\right)=\left(x_{1}, \ldots, x_{i-1}, y_{i}, x_{i+1}, \ldots, x_{l}\right)$. The usual noncooperative game corresponds to the case $D=X$, then (2) is equivalent to the well known Nash equilibrium concept. Examples of applications of generalized noncooperative games with joint constraints can be e.g. found in [6, 9, 17, 10, 11]. The main question consists in implementation of this constrained equilibrium concept within the custom noncooperative framework, where players are independent and make their choices simultaneously. In fact, the presence of the binding constraints requires certain treaties or concordant actions of the players, thus contradicting the above assumptions. These drawbacks were noticed and discussed e.g. in [5, 12, 8].

Rather recently, the right-hand side decomposition approach was suggested for variational inequalities with binding constraints in [13. Its extension to generalized noncooperative games was given in [11]. Within this approach, the initial problem is treated as a two-level one by using a share allocation procedure, which leads to a set-valued variational inequality as a master problem. In [11], a usual noncooperative game problem is solved at the lower level. Further, a decomposable dual regularization (penalty) method that deals with a single-valued approximation of the master problem for each fixed share allocation was suggested. Application of this method for production problems with common pollution regulation was described in [14].

In this paper, we suggest to apply a direct decomposable penalty method to generalized noncooperative games, which involve share allocation variables. We show convergence of solutions of auxiliary penalized problems to a solution of the initial problem under weak coercivity conditions. Besides, the implementation of this method enables one to solve each auxiliary penalized problem as a two-level one with the usual Nash equilibrium problem at the lower level.

\section{Decomposition via Shares Allocation}

We first fix our basic assumptions.

(A1) Each strategy set $X_{i} \subseteq \mathbb{R}^{n_{i}}$ is convex and closed and each utility function $f_{i}$ is concave in its $i$-th variable $x_{i}$ and continuous for $i=1, \ldots, l$. Also, $h_{i j}: \mathbb{R}^{n_{i}} \rightarrow \mathbb{R}$,

$j=1, \ldots, m, i=1, \ldots, l$ are convex functions, and the common feasible set $D$ is nonempty.

Following the Nikaido-Isoda approach from [15], we consider the normalized equilibrium problem (EP for short) of finding a point $x^{*}=\left(x_{1}^{*}, \ldots, x_{l}^{*}\right)^{\top} \in D$ such that

$$
\Phi\left(x^{*}, y\right) \geq 0 \quad \forall y \in D,
$$


where

$$
\Phi(x, y)=\Psi(x, x)-\Psi(x, y), \Psi(x, y)=\sum_{i=1}^{l} f_{i}\left(x_{-i}, y_{i}\right)
$$

its solutions are called normalized equilibrium points. From the above assumptions it follows that $\Phi: X \times X \rightarrow \mathbb{R}$ is an equilibrium bi-function, i.e., $\Phi(x, x)=0$ for every $x \in X$, besides, $\Phi(x, \cdot)$ is convex for each $x \in X$ and $\Phi(\cdot, \cdot)$ is continuous. It should be noted that (3) implies (2). In other words, each normalized equilibrium point is a generalized Nash equilibrium point, but the reverse assertion is not true in general. But in case $D=X,(2)$ and (3) become equivalent. We take a suitable coercivity condition and obtain the existence result from [16, Theorem 3.1].

For a function $\mu: \mathbb{R}^{n} \rightarrow \mathbb{R}$ and a number $r$, we define the level set

$$
B_{r}=\{x \in X \mid \mu(x) \leq r\} .
$$

We say that the function $\mu: \mathbb{R}^{n} \rightarrow \mathbb{R}$ is weakly coercive with respect to the set $X \subseteq \mathbb{R}^{n}$ if there exists a number $r$ such that the set $B_{r}$ is nonempty and bounded.

(C1) There exist a lower semicontinuous and convex function $\mu: X \rightarrow \mathbb{R}$, which is weakly coercive with respect to the set $D$, and a number $r$, such that, for any point $x \in D \backslash B_{r}$ there is a point $z \in D$ with

$$
\min \{\Phi(x, z), \mu(z)-\mu(x)\}<0 \quad \text { and } \quad \max \{\Phi(x, z), \mu(z)-\mu(x)\} \leq 0 .
$$

Proposition 1 If (A1) and (C1) are fulfilled, then problem (3) has a solution.

After proper specialization of the inequalities in (C1) we can somewhat strengthen the above assertion.

(C2) There exist a lower semicontinuous and convex function $\mu: X \rightarrow \mathbb{R}$, which is weakly coercive with respect to the set $D$, and a number $r$, such that, for any point $x \in D \backslash B_{r}$ there is a point $z \in D$ with

$$
\mu(z) \leq \mu(x) \quad \text { and } \Phi(x, z)<0 .
$$

Corollary 1 If (A1) and (C2) are fulfilled, then problem (3) has a solution, and all the solutions are contained in $D \bigcap B_{r}$.

These coercivity conditions (C1) and (C2) clearly hold if $D($ or $X)$ is bounded. Then we can take $\mu(x)=\|x\|$ and choose $r$ large enough so that $D \subset B_{r}$.

Let us now introduce the set of partitions of the right-hand side common constraint vector $b$ :

$$
\tilde{U}=\left\{u \in \mathbb{R}^{m l} \mid \sum_{i=1}^{l} u_{i}=b\right\} .
$$

where $u=\left(u_{1}, \ldots, u_{l}\right)^{\top}, u_{i} \in \mathbb{R}^{m}, i=1, \ldots, l$. Here $u_{i}$ determines the share of the $i$-th player. 
Given a partition $u \in \tilde{U}$, we can consider the parametric EP: Find a point $x(u)=$ $\left(x_{1}(u), \ldots, x_{l}(u)\right)^{\top} \in D(u)$ such that

$$
\Phi\left(x^{*}, y\right) \geq 0 \quad \forall y \in D(u)
$$

where

$$
D(u)=D_{1}\left(u_{1}\right) \times \ldots \times D_{l}\left(u_{l}\right),
$$

$D_{i}\left(u_{i}\right)=\left\{x_{i} \in X_{i} \mid h_{i}\left(x_{i}\right) \leq u_{i}\right\}, i=1, \ldots, l$. Clearly, (4) is equivalent to the parametric Nash equilibrium problem (NEP):

$$
f_{i}\left(x_{-i}(u), y_{i}\right) \leq f_{i}(x(u)) \quad \forall y_{i} \in D_{i}\left(u_{i}\right), i=1, \ldots, l .
$$

If all the optimal shares $u_{i}, i=1, \ldots, l$ of players are known, the constrained problems (2) and (3) reduce to NEPs. Hence, it seems worthwhile to insert an additional upper control level for finding the optimal shares. In optimization, this approach is known as the right-hand side (Kornai-Liptak) decomposition method; see [17].

Following this approach we notice that under certain regularity condition system (5) can be replaced with the corresponding system of primal-dual optimality conditions: Find a pair $(x(u), v(u)) \in X \times \mathbb{R}_{+}^{m l}$ such that

$$
\begin{array}{ll}
f_{i}(x(u))-f_{i}\left(x_{-i}(u), y_{i}\right) & \\
+\left\langle v_{i}(u), h_{i}\left(y_{i}\right)-h_{i}\left(x_{i}(u)\right)\right\rangle \geq 0 & \forall y_{i} \in X_{i}, \\
\left\langle u_{i}-h_{i}\left(x_{i}(u)\right), v_{i}-v_{i}(u)\right\rangle \geq 0, & \forall v_{i} \in \mathbb{R}_{+}^{m}, \\
\text { for } i=1, \ldots, l &
\end{array}
$$

where $v(u)=\left(v_{1}(u), \ldots, v_{l}(u)\right)^{\top}$. In this system the first relations (6) are rewritten equivalently as

$$
\begin{aligned}
& \Phi(x(u), y) \\
& +\sum_{i=1}^{l}\left\langle v_{i}(u), h_{i}\left(y_{i}\right)-h_{i}\left(x_{i}(u)\right)\right\rangle \geq 0 \quad \forall y \in X .
\end{aligned}
$$

Clearly, if $(x(u), v(u))$ solves (6) -(17) or (8) , (17), then $x(u)$ is a solution to (44) or (5)).

We denote by $T(u)$ the set of all the solution points $-v(u)$, creating the image of the set-valued mapping $T$. This enables us to define the variational inequality (VI): Find a point $u^{*} \in \tilde{U}$ such that

$$
\exists t^{*} \in T\left(u^{*}\right),\left\langle t^{*}, u-u^{*}\right\rangle \geq 0, \quad \forall u \in \tilde{U} .
$$

Then it was shown in [11, Theorem 4.1] that just the master VI (9) yields the optimal shares of common constraints among players.

Proposition 2 Suppose (A1) is fulfilled. If a point $u^{*}$ solves VI (9), the corresponding point $x\left(u^{*}\right)$ in (6) - (7) is a solution of problem (1), (3). 
We conclude that VI (9) related to the parametric problems (4) or (15) enables us to find a solution to the initial generalized noncooperative game. Hence, the two-level procedure gives a suitable regulation mechanism for these game problems. However, this approach has clear drawbacks: $T(u)$ can be empty for some feasible partitions, besides, $T$ is set-valued in general, and this fact reduces the number of methods applicable to solution of VI (9). Therefore, this approach needs certain modifications.

\section{Decomposable Penalty Method}

We start our description of the approach from the simple transformation of the joint constraint set $Y$ by inserting auxiliary variables:

$$
Y=\left\{x \in \mathbb{R}^{n} \mid \exists u \in \mathbb{R}^{m l}, \sum_{i=1}^{l} u_{i}=b, h_{i}\left(x_{i}\right) \leq u_{i}, i=1, \ldots, l\right\},
$$

where $u=\left(u_{1}, \ldots, u_{l}\right)^{\top}, u_{i} \in \mathbb{R}^{m}, i=1, \ldots, l$. These variables $u_{i}$ as above determine a partition of the right-hand side vector $b$, i.e. give explicit shares of players. In principle, some additional reasonable restrictions can be imposed on the shares $u$, such as $u_{i} \leq b$ or $/$ and $u_{i} \geq \mathbf{0}$ for $i=1, \ldots, l$. Hence, we define the set of feasible partitions as follows:

$$
U=\left\{u \in U_{0} \mid \sum_{i=1}^{l} u_{i}=b\right\}
$$

where $U_{0} \subseteq \mathbb{R}^{m l}$ is a set of these optional additional restrictions such that for each $x \in D$ there exists $u \in U, h_{i}\left(x_{i}\right) \leq u_{i}, i=1, \ldots, l$.

We can now separate the constraints and first consider the auxiliary penalty problem: Find a pair $w(\tau)=(x(\tau), u(\tau)) \in X \times U, \tau>0$ such that

$$
\Phi_{\tau}(w(\tau), w)=\Phi(x(\tau), x)+\tau[P(w)-P(w(\tau))] \geq 0 \quad \forall w=(x, u) \in X \times U,
$$

where

$$
P(w)=\sum_{i=1}^{l} P_{i}\left(w_{i}\right)
$$

and each $P_{i}\left(w_{i}\right)=P_{i}\left(x_{i}, u_{i}\right)$ is a general penalty function for the set

$$
W_{i}=\left\{w_{i}=\left(x_{i}, u_{i}\right) \in \mathbb{R}^{n_{i}} \times \mathbb{R}^{m} \mid h_{i}\left(x_{i}\right) \leq u_{i}\right\}
$$

We will define these functions as follows:

$$
P_{i}\left(x_{i}, u_{i}\right)=\varphi\left(h_{i}\left(x_{i}\right)-u_{i}\right), \quad i=1, \ldots, l,
$$


where $\varphi: \mathbb{R}^{m} \rightarrow \mathbb{R}_{+}$is a convex differentiable and isotone function such that

$$
\varphi(v) \begin{cases}=0, & \text { if } v \leq \mathbf{0} \\ >0, & \text { otherwise. }\end{cases}
$$

It follows that

$$
P_{i}\left(x_{i}, u_{i}\right) \begin{cases}=0, & \left(x_{i}, u_{i}\right) \in W_{i} \\ >0, & \left(x_{i}, u_{i}\right) \notin W_{i}\end{cases}
$$

for $i=1, \ldots, l$. We recall that the function $\varphi: \mathbb{R}^{m} \rightarrow \mathbb{R}$ is called isotone, if for any points $u, v, u \geq v$ it holds that $\varphi(u) \geq \varphi(v)$. The most popular and simple choice is

$$
\varphi(v)=0.5\left\|[v]_{+}\right\|^{2}
$$

where $[v]_{+}$denotes the projection of $v$ onto the non-negative orthant $\mathbb{R}_{+}^{m}$. Then each penalty function $P_{i}$ is convex and differentiable for $i=1, \ldots, l$. We observe that decomposable penalty methods were suggested for separable convex optimization problems in [18, 19].

We now show that problem (10) can be replaced with the following: Find a pair $w(\tau)=(x(\tau), u(\tau)) \in X \times U$, such that

$$
\begin{aligned}
& \Phi(x(\tau), x)+\tau \sum_{i=1}^{l}\left\langle\varphi^{\prime}\left(h_{i}\left(x_{i}(\tau)\right)-u_{i}(\tau)\right), h_{i}\left(x_{i}\right)-h_{i}\left(x_{i}(\tau)\right)\right\rangle \\
& +\tau \sum_{i=1}^{l}\left\langle\varphi^{\prime}\left(h_{i}\left(x_{i}(\tau)\right)-u_{i}(\tau)\right), u_{i}(\tau)-u_{i}\right\rangle \geq 0 \quad \forall w=(x, u) \in X \times U .
\end{aligned}
$$

Let $w(\tau)$ solve (11). Then (10) holds due to the convexity of the function $\varphi$. In fact, we have

$$
\begin{aligned}
P_{i}\left(x_{i}, u_{i}\right)-P_{i}\left(x_{i}(\tau), u_{i}(\tau)\right) \geq \quad & \left\langle\varphi^{\prime}\left(h_{i}\left(x_{i}(\tau)\right)-u_{i}(\tau)\right), h_{i}\left(x_{i}\right)-h_{i}\left(x_{i}(\tau)\right)\right\rangle \\
& -\left\langle\varphi^{\prime}\left(h_{i}\left(x_{i}(\tau)\right)-u_{i}(\tau)\right), u_{i}-u_{i}(\tau)\right\rangle,
\end{aligned}
$$

for $i=1, \ldots, l$, and (11) implies (10).

Conversely, let $w(\tau)=(x(\tau), u(\tau))$ be a solution of problem (10). Then we can temporarily set $\phi(x)=\Phi(x(\tau), x)$ and obtain

$$
\phi(x)-\phi(x(\tau))+\tau[P(w)-P(w(\tau))] \geq 0 \quad \forall w=(x, u) \in X \times U,
$$

i.e. $w(\tau)$ is a solution of the optimization problem. Applying now Proposition 4 in [20] we conclude that $w(\tau)$ solves (11).

In turn, problem (11) is clearly equivalent to the system: Find a pair $w(\tau)=$ $(x(\tau), u(\tau)) \in X \times U$, such that

$$
\begin{aligned}
& \Phi(x(\tau), x)+\tau \sum_{i=1}^{l}\left\langle\varphi^{\prime}\left(h_{i}\left(x_{i}(\tau)\right)-u_{i}(\tau)\right), h_{i}\left(x_{i}\right)-h_{i}\left(x_{i}(\tau)\right)\right\rangle \geq 0 \quad \forall x \in X, \\
& \sum_{i=1}^{l}\left\langle\varphi^{\prime}\left(h_{i}\left(x_{i}(\tau)\right)-u_{i}(\tau)\right), u_{i}(\tau)-u_{i}\right\rangle \geq 0 \quad \forall u \in U
\end{aligned}
$$


We now collect the obtained properties.

Lemma 1 Let the conditions in (A1) be fulfilled. Then problems (10), (11), and (12)-(13) are equivalent.

Given a point $u \in \mathbb{R}^{m l}$, we can solve only problem (12) in $x$, which is to find $x(u) \in X$ such that

$$
\Phi(x(u), x)+\tau \sum_{i=1}^{l}\left\langle\varphi^{\prime}\left(h_{i}\left(x_{i}(u)\right)-u_{i}\right), h_{i}\left(x_{i}\right)-h_{i}\left(x_{i}(u)\right)\right\rangle \geq 0 \quad \forall x \in X .
$$

Let $X(u)$ denote the whole solution set of this problem. For each $x(u) \in X(u)$ we set

$$
g(u)=\left(g_{1}(u), \ldots, g_{l}(u)\right)^{\top}, \text { where } g_{i}(u)=-\varphi^{\prime}\left(h_{i}\left(x_{i}(u)\right)-u_{i}\right), \quad i=1, \ldots, l .
$$

Thus we can define the mapping value

$$
G(u)=\{g(u) \mid x(u) \in X(u)\} .
$$

Bearing in mind (13), we now define the VI: Find a point $u^{*} \in U$ such that

$$
\exists g\left(u^{*}\right) \in G\left(u^{*}\right),\left\langle g\left(u^{*}\right), u-u^{*}\right\rangle \geq 0, \quad \forall u \in U .
$$

Proposition 3 Suppose (A1) is fulfilled.

(i) If a point $u^{*}$ solves VI (16), then there exists a point $x^{*}=x\left(u^{*}\right) \in X\left(u^{*}\right)$ such that $g\left(u^{*}\right)$ is defined in (15) at $u=u^{*}$ and that the pair $w^{*}=\left(x^{*}, u^{*}\right)$ is a solution of problem (10).

(ii) If a pair $w(\tau)=(x(\tau), u(\tau))$ is a solution of problem (10), then the point $u^{*}=u(\tau)$ solves $V I(16)$.

The assertions follow directly from the definitions and Lemma 1.

The next step consists in replacing problem (14) with the following penalized EP: Find $x(u) \in X$ such that

$$
\Phi(x(u), y)+\tau \sum_{i=1}^{l}\left(P_{i}\left(y_{i}, u_{i}\right)-P_{i}\left(x_{i}(u), u_{i}\right)\right) \geq 0 \quad \forall y \in X .
$$

The equivalence of (14) and (17) is proved similarly to Lemma 1, However, (17) is clearly equivalent to the NEP: Find $x(u) \in X$ such that

$$
\tilde{f}_{i}\left(x_{-i}(u), y_{i}\right) \leq \tilde{f}_{i}(x(u)), \quad \forall y_{i} \in X_{i}, i=1, \ldots, l ;
$$

where the $i$-th player has the penalized utility function

$$
\tilde{f}_{i}(x)=f_{i}(x)-\tau P_{i}\left(x_{i}, u_{i}\right)
$$

cf. (2) . Therefore, $X(u)$ is now also the whole solution set of NEP (18)-(19) and we have obtained the basic equivalence result. 
Theorem 1 Suppose (A1) is fulfilled.

(i) If a point $u^{*}$ solves VI (16), then there exists a solution $x^{*}=x\left(u^{*}\right)$ of NEP (18)-(19) and $g\left(u^{*}\right)$ is defined in (15) at $u=u^{*}$, such that the pair $w^{*}=\left(x^{*}, u^{*}\right)$ is a solution of problem (10).

(ii) If a pair $w(\tau)=(x(\tau), u(\tau))$ is a solution of problem (10), then the point $u^{*}=u(\tau)$ solves $V I(16)$ and the point $x(\tau)$ is a solution of NEP (18)-(19) at $u=u(\tau)$.

We conclude that VI (16) related to the parametric NEP (18)-(19) yields a solution for the penalized game problem. Hence, we have derived another two-level decomposition method for the initial generalized noncooperative game and have to indicate its preferences over the method of Section 2, First of all we observe that NEP (18)-(19) has a solution under rather mild assumptions. From Proposition 1 we obtain that this is the case if (A1) holds and the set $X$ is bounded. Also, we can take a suitable coercivity condition in the unbounded case.

(C3) There exists a point $\tilde{x} \in X$ such that

$$
\Phi\left(x^{k}, \tilde{x}\right) \rightarrow-\infty \quad \text { as } \quad\left\|x^{k}-\tilde{x}\right\| \rightarrow \infty ;
$$

for any infinite sequence $\left\{x^{k}\right\} \subset X$.

Proposition 4 If (A1) and (C3) are fulfilled, then NEP (18)-(19) has a solution for each $\tau>0$.

Proof. It suffices to show that problem (17) has a solution. Set $\mu(x)=\|x-\tilde{x}\|$ and

$$
\tilde{\Phi}(x, y)=\Phi(x, y)+\tau[P(y, u)-P(x, u)]
$$

for a fixed $u \in U$. Then

$$
\tilde{\Phi}\left(x^{k}, \tilde{x}\right) \leq \Phi\left(x^{k}, \tilde{x}\right)+\tau P(\tilde{x}, u) \rightarrow-\infty \quad \text { as } \quad\left\|x^{k}-\tilde{x}\right\| \rightarrow \infty .
$$

This means that (C3) implies (C1) for problem (17) and the result follows from Proposition 1,

For this reason, $G(u)$ is non-empty under usual assumptions even if the set $D(u)$ is empty. The other preference is that $G$ possesses a strengthened monotonicity property.

Proposition 5 Suppose (A1) is fulfilled, the bi-function $\Phi$ is monotone on $X \times X$, i.e.

$$
\Phi\left(x^{\prime}, x^{\prime \prime}\right)+\Phi\left(x^{\prime \prime}, x^{\prime}\right) \leq 0 ;
$$

for each pair of points $x^{\prime}, x^{\prime \prime} \in X$, and the gradientmap $\varphi^{\prime}$ is co-coercive with constant $\gamma$, i.e.

$$
\left\langle v^{\prime}-v^{\prime \prime}, \varphi^{\prime}\left(v^{\prime}\right)-\varphi^{\prime}\left(v^{\prime \prime}\right)\right\rangle \geq \gamma\left\|\varphi^{\prime}\left(v^{\prime}\right)-\varphi^{\prime}\left(v^{\prime \prime}\right)\right\|^{2}
$$

for all $v^{\prime}, v^{\prime \prime} \in \mathbb{R}^{m l}$. Then the mapping $G$ is co-coercive with constant $\gamma$. 
Proof. Take arbitrary points $u^{\prime}, u^{\prime \prime} \in \mathbb{R}^{m l}$ and set $x^{\prime}=x\left(u^{\prime}\right), g^{\prime}=g\left(u^{\prime}\right), v^{\prime}=h\left(x^{\prime}\right)-u^{\prime}$ and $x^{\prime \prime}=x\left(u^{\prime \prime}\right), g^{\prime \prime}=g\left(u^{\prime \prime}\right), v^{\prime \prime}=h\left(x^{\prime \prime}\right)-u^{\prime \prime}$. It follows from (14) that

$$
\begin{aligned}
& \Phi\left(x^{\prime}, x^{\prime \prime}\right)-\tau \sum_{i=1}^{l}\left\langle g_{i}^{\prime}, h_{i}\left(x_{i}^{\prime \prime}\right)-h_{i}\left(x_{i}^{\prime}\right)\right\rangle \geq 0, \\
& \Phi\left(x^{\prime \prime}, x^{\prime}\right)-\tau \sum_{i=1}^{l}\left\langle g_{i}^{\prime \prime}, h_{i}\left(x_{i}^{\prime}\right)-h_{i}\left(x_{i}^{\prime \prime}\right)\right\rangle \geq 0
\end{aligned}
$$

hence

$$
\sum_{i=1}^{l}\left\langle g_{i}^{\prime}-g_{i}^{\prime \prime}, h_{i}\left(x_{i}^{\prime}\right)-h_{i}\left(x_{i}^{\prime \prime}\right)\right\rangle \geq-\left[\Phi\left(x^{\prime}, x^{\prime \prime}\right)+\Phi\left(x^{\prime \prime}, x^{\prime}\right)\right] / \tau \geq 0
$$

since $\Phi$ is monotone. It follows that

$$
\begin{aligned}
& 0 \leq \sum_{i=1}^{l}\left\langle g_{i}^{\prime}-g_{i}^{\prime \prime},\left[h_{i}\left(x_{i}^{\prime}\right)-u_{i}^{\prime}\right]-\left[h_{i}\left(x_{i}^{\prime \prime}\right)-u_{i}^{\prime \prime}\right]\right\rangle+\sum_{i=1}^{l}\left\langle g_{i}^{\prime}-g_{i}^{\prime \prime}, u_{i}^{\prime}-u_{i}^{\prime \prime}\right\rangle \\
& =-\sum_{i=1}^{l}\left\langle\varphi^{\prime}\left(v_{i}^{\prime}\right)-\varphi^{\prime}\left(v_{i}^{\prime \prime}\right), v_{i}^{\prime}-v_{i}^{\prime \prime}\right\rangle+\left\langle g^{\prime}-g^{\prime \prime}, u^{\prime}-u^{\prime \prime}\right\rangle \\
& \leq-\gamma \sum_{i=1}^{l}\left\|\varphi^{\prime}\left(v_{i}^{\prime}\right)-\varphi^{\prime}\left(v_{i}^{\prime \prime}\right)\right\|^{2}+\left\langle g^{\prime}-g^{\prime \prime}, u^{\prime}-u^{\prime \prime}\right\rangle \\
& =-\gamma\left\|g^{\prime}-g^{\prime \prime}\right\|^{2}+\left\langle g^{\prime}-g^{\prime \prime}, u^{\prime}-u^{\prime \prime}\right\rangle,
\end{aligned}
$$

therefore, $G$ is co-coercive with constant $\gamma$.

It is well-known that the gradientmap of a convex differentiable function is cocoercive with constant $\gamma$ if it satisfies the Lipschitz condition with constant $1 / \gamma$; see e.g. [21, Chapter I, Lemma 6.7]. It follows that the mapping $G$ is then single-valued even if this is not the case for the mapping $u \mapsto X(u)$. The assertion of Proposition 5 holds true if we replace the monotonicity of the bi-function $\Phi$ with the more general monotonicity property of the mapping $F$ defined by taking the sub-differential in $y$ for $\Phi(x, y)$, i.e.,

$$
F(x)=\left.\frac{\partial \Phi(x, y)}{\partial y}\right|_{y=x} ;
$$

and following the lines of Proposition 7.2 in [11]. Proposition [5 shows that VI (16) admits more efficient solution methods in comparison with VI (9); see e.g. [11, 21].

We observe that the equivalent transformations from (10) to (11) and (16)-(17) are crucial for the decomposable penalty method. The solution concept based on (16)-(17) has a rather simple and natural interpretation. A system regulator chooses first the penalty parameter $\tau$. Afterwards, he/she determines the right share allocation vector $u(\tau)$ by sending some trial vectors $u$ to players and announcing particular deviation 
penalty functions, the players then make proper corrections of their utility functions and determine the corresponding Nash equilibrium point for each trial vector. Then the system regulator changes the penalty parameter etc. Application of the other known iterative solution methods to the above generalized game problems was analyzed in [11. They include in particular the usual penalty methods. It appeared that implementation of these mechanisms within a noncooperative game framework may meet serious difficulties; see [11] for more details.

\section{Convergence of the Penalty Method}

In this section, we intend to substantiate the penalty method with the auxiliary problem (10) (or (11)). First we take the following coercivity condition for EP (10).

(C4) There exist a lower semicontinuous and convex function $\eta: X \times U \rightarrow \mathbb{R}$, which is weakly coercive with respect to the set $X \times U$, and a number $r$, such that, for any point $w=(x, u) \in(X \times U)$ such that $\eta(w)>r$ there is a point $w^{\prime}=(z, v) \in(X \times U)$ with

$$
\eta\left(w^{\prime}\right) \leq \eta(w) \quad \text { and } \quad \Phi_{\tau}\left(w, w^{\prime}\right)<0 .
$$

We note that (C4) is a clear adjustment of condition (C2). For brevity, we define the level set

$$
E_{r}=\{w=(x, u) \in(X \times U) \mid \eta(w) \leq r\} .
$$

Lemma 2 Let the conditions in (A1) and (C2) be fulfilled for some $\tau>0$. Then problem (10) has a solution, and all the solutions are contained in $(X \times U) \bigcap E_{r}$.

The assertion follows from Corollary 1 .

However, condition (C4) is not suitable for verification (cf. (C3)) and we will deduce it from other conditions of form (C2). In general, we follow the approach from [16]. First we consider the case where the set $U$ is bounded.

(C5) There exist a lower semicontinuous and convex function $\mu: X \rightarrow \mathbb{R}$, which is weakly coercive with respect to the set $X$, and a number $r$, such that, for any point $x \in X \backslash B_{r}$ there is a point $z \in D$ with

$$
\mu(z) \leq \mu(x) \quad \text { and } \Phi(x, z)<0 .
$$

Theorem 2 Suppose that (A1) and (C5) are fulfilled, the set $U$ is bounded, the sequence $\left\{\tau_{k}\right\}$ satisfies

$$
\left\{\tau_{k}\right\} \nearrow+\infty
$$

Then:

(i) EP (3) has a solution;

(ii) $E P(10)$ has a solution for each $\tau>0$ and all these solutions belong to $B_{r} \times U$;

(iii) Each sequence $\left\{w\left(\tau_{k}\right)\right\}$ of solutions of EP (10) has limit points, all these limit points belong to $\left(B_{r} \cap D\right) \times U$ and are solutions of $E P$ (3). 
Proof. We first show that, for any $\tau>0,(\mathbf{C} 4)$ is true with $\eta(w)=\mu(x)$. Take any $w=(x, u) \in(X \times U) \backslash E_{r}$, then by (C5) there is $z \in D, \mu(z) \leq \mu(x)$ such that $\Phi(x, z)<0$. Since $z \in D$, there exists $v \in U$ such that $P\left(w^{\prime}\right)=P(z, v)=0$ and we have

$$
\Phi_{\tau}\left(w, w^{\prime}\right)=\Phi(x, z)+\tau\left[P\left(w^{\prime}\right)-P(w)\right] \leq \Phi(x, z)-\tau P(w)<0 .
$$

Hence, assertion (ii) follows from Lemma 2 ,

For brevity, we set $w^{k}=w\left(\tau_{k}\right)=\left(x^{k}, u^{k}\right)$, where $x^{k}=x\left(\tau_{k}\right)$ and $u^{k}=u\left(\tau_{k}\right)$. By (ii), the sequence $\left\{w^{k}\right\}$ exists and is bounded. Therefore, it has limit points. Since $B_{r}$ is convex and closed, all these limit points must belong to $B_{r} \times U$. Let $\bar{w}=(\bar{x}, \bar{u})$ be an arbitrary limit point of $\left\{w^{k}\right\}$, i.e. $\left\{w^{k_{s}}\right\} \rightarrow \bar{w}$. Then, by definition,

$$
0 \leq P\left(w^{k_{s}}\right) \leq \tau_{k_{s}}^{-1} \Phi\left(x^{k_{s}}, x\right)+P(x, u), \quad \forall(x, u) \in X \times U .
$$

Taking $(x, u) \in D \times U$ such that $P(x, u)=0$ and using (20), we obtain

$$
0 \leq P(\bar{w}) \leq \liminf _{s \rightarrow \infty} P\left(w^{k_{s}}\right) \leq \limsup _{s \rightarrow \infty}\left[\tau_{k_{s}}^{-1} \Phi\left(x^{k_{s}}, x\right)\right] \leq 0,
$$

i.e. $P(\bar{w})=0$ and $\bar{x} \in D$. Therefore, for each $x \in D$ we can take $u \in U$ such that $P(w)=P(x, u)=0$ and obtain

$$
\Phi\left(x^{k_{s}}, x\right)-\tau_{k_{s}} P\left(w^{k_{s}}\right)=\Phi\left(x^{k_{s}}, x\right)+\tau_{k_{s}}\left[P(w)-P\left(w^{k_{s}}\right)\right] \geq 0 .
$$

It now follows that

$$
\Phi(\bar{x}, x) \geq \limsup _{s \rightarrow+\infty} \Phi\left(x^{k_{s}}, x\right) \geq \limsup _{s \rightarrow+\infty}\left[\tau_{k_{s}} P\left(x^{k_{s}}\right)\right] \geq 0 .
$$

Therefore $\bar{x}$ solves EP (3) and assertion (iii) is true. Since $\bar{x}$ exists, assertion (i) is also true. The proof is complete.

We now give similar properties in the unbounded case.

Theorem 3 Suppose that (A1) and (C5) are fulfilled, the sequence $\left\{\tau_{k}\right\}$ satisfies condition (20). Then:

(i) EP (3) has a solution;

(ii) There exists $\tau^{\prime}>0$ such that EP (10) has a solution for each $\tau>\tau^{\prime}$ and all these solutions belong to $E_{r} \bigcap(X \times U)$ where $\eta(w)=\max \{\mu(x), P(w)\}$;

(iii) Each sequence $\left\{w\left(\tau_{k}\right)\right\}$ of solutions of EP (10) has limit points, all these limit points belong to $\left(B_{r} \cap D\right) \times U$ and are solutions of $E P$ (3).

Proof. We first show that the function $\eta(w)=\max \{\mu(x), P(w)\}$ is weakly coercive with respect to the set $X \times U$. Fix a number $r$ such that the set $E_{r} \bigcap(X \times U)$ is nonempty. If it is unbounded, there exists a sequence $\left\{w^{k}\right\}$ such that $w^{k}=\left(x^{k}, u^{k}\right) \in$ $E_{r} \bigcap(X \times U)$ such that $\left\|w^{k}\right\| \rightarrow \infty$ as $k \rightarrow \infty$. Since the function $\mu(x)$ is weakly coercive with respect to the set $X$, we have $\left\|x^{k}\right\| \leq C \leq \infty$. It follows that $\left\|u^{k}\right\| \rightarrow \infty$. 
Since $u \in U$, there exists at least one pair of indices $j$ and $t$ such that $u_{j t}^{k_{s}} \rightarrow-\infty$ for the corresponding subsequence $\left\{u^{k_{s}}\right\}$, hence $P_{j}\left(x_{j}^{k_{s}}, u_{j}^{k_{s}}\right) \rightarrow+\infty$, which is a contradiction.

We now show that there exists $\tau^{\prime}>0$ such that $(\mathbf{C} 4)$ is true for any $\tau>\tau^{\prime}$. Take any $w=(x, u) \in(X \times U) \backslash E_{r}$, then $\eta(w)>r$. If $\mu(x)>r$, then by (C5) there is $z \in D, \mu(z) \leq \mu(x)$ such that $\Phi(x, z)<0$. Since $z \in D$, there exists $v \in U$ such that $P\left(w^{\prime}\right)=P(z, v)=0$ and we have

$$
\Phi_{\tau}\left(w, w^{\prime}\right)=\Phi(x, z)+\tau\left[P\left(w^{\prime}\right)-P(w)\right] \leq \Phi(x, z)-\tau P(w)<0 .
$$

Hence, (C4) holds. If $\mu(x) \leq r$, then $P(w)>r$. Fix a point $z \in D$, then there exists $v \in U$ such that $P\left(w^{\prime}\right)=P(z, v)=0$. Since the set $B_{r}$ is bounded, we have

$$
\max _{x \in B_{r}} \Phi(x, z)=d<\infty .
$$

Take any $\tau^{\prime}=d / r$, then

$$
\Phi_{\tau}\left(w, w^{\prime}\right)=\Phi(x, z)+\tau\left[P\left(w^{\prime}\right)-P(w)\right] \leq d-\tau r<0
$$

if $\tau>\tau^{\prime}$. Hence, (C4) also holds and assertion (ii) follows from Lemma 2, Assertions (iii) and (i) are proved as in Theorem 2.

\section{References}

[1] Debreu G (1952) A social equilibrium existence theorem. Proc Nat Acad Sci USA 38: $886-893$

[2] Rosen JB (1965) Existence and uniqueness of equilibrium points for concave Nperson games. Econometrica 33: 520-533

[3] Zukhovitskii SI, Polyak RA, Primak ME (1969) Two methods of search for equilibrium points of $n$-person concave games. Soviet Mathem Dokl 10: 279-282

[4] Zukhovitskii SI, Polyak RA, Primak ME (1971) Concave many person games. Ekon Matem Metody 7: 888-900 (in Russian)

[5] Ichiishi T (1983) Game theory for economic analysis. Academic Press, New York

[6] Krawczyk JB, Uryasev S (2000) Relaxation algorithms to find Nash equilibria with economic applications. Environ Model Assess 5: 63-73

[7] Facchinei F, Kanzow C (2007) Generalized Nash equilibrium problems. 4OR 5: $173-210$

[8] Fukushima M (2011) Restricted generalized Nash equilibria and controlled penalty algorithm. Comput Manag Sci 8: 201-218 
[9] Contreras J, Klusch M, Krawczyk JB (2004) Numerical solutions to Nash-Cournot equilibria in coupled constraint electricity markets. IEEE Trans Power Syst 19: 195-206

[10] Pang J-S, Scutari G, Facchinei F, Wang C (2008) Distributed power allocation with rate constraints in Gaussian parallel interference channels. IEEE Trans Inform Theory 54: 3471-3489

[11] Konnov IV (2016) Shares allocation methods for generalized game problems with joint constraints. Set-Valued Variat Anal 24: 499-516

[12] Harker PT (1991) Generalized Nash games and quasivariational inequalities. Eur J Oper Res 54: 81-94

[13] Konnov IV (2014) Right-hand side decomposition for variational inequalities. J Optim Theory Appl 160: 221-238

[14] Allevi E., Gnudi A., Konnov I.V., Oggioni G. (2018) Decomposition method for oligopolistic competitive models with common environmental regulation. Ann Oper Res 268: 441-467

[15] Nikaido H, Isoda K (1955) Note on noncooperative convex games. Pacific J Mathem 5: 807-815

[16] Konnov I.V. (2015) Regularized penalty method for general monotone equilibrium problems in Banach spaces. J Optim Theory Appl 164: 500-513

[17] Kornai J, Liptak T (1965) Two-level planning. Econometrica 33: 141-169

[18] Razumikhin BS (1967) Iterative method for the solution and decomposition of linear programming problems. Autom Remote Control 29: 427-443

[19] Umnov AE (1975) The method of penalty functions in problems of large dimension. USSR Comp Maths Math Phys 15: 32-45

[20] Konnov I.V. (2019) An approximate penalty method with descent for convex optimization problems. Russ.Mathem. (Iz. VUZ) 63(7): 41-55

[21] Gol'shtein, E.G., Tret'yakov, N.V. (1989) Augmented Lagrange functions. Nauka, Moscow (Engl. transl. in John Wiley and Sons, New York, 1996) 